\title{
Adhesiolysis for Liver Resection in a Patient with a Reconstructed Stomach Tube after Esophagectomy
}

\author{
Shigeru Fujisaki ${ }^{1,2}$, Motoi Takashina', Kenichi Sakurai ${ }^{3}$, \\ Ryouichi Tomita ${ }^{3}$, Tadatoshi Takayama ${ }^{2}$ \\ ${ }^{1}$ Department of Surgery, Fujisaki Hospital, Tokyo, Japan \\ ${ }^{2}$ Division of Digestive Surgery, Nihon University School of Medicine, Tokyo, Japan \\ ${ }^{3}$ Department of Surgery, Nippon Dental University, Tokyo, Japan \\ Email: ${ }^{\star}$ sfujisak@blue.ocn.ne.jp
}

How to cite this paper: Fujisaki, S., Takashina, M., Sakurai, K., Tomita, R. and Takayama, T. (2020) Adhesiolysis for Liver Resection in a Patient with a Reconstructed Stomach Tube after Esophagectomy. Journal of Cancer Therapy, 11, 371-376. https://doi.org/10.4236/jct.2020.116032

Received: May 21, 2020

Accepted: June 8, 2020

Published: June 11, 2020

Copyright $\odot 2020$ by author(s) and Scientific Research Publishing Inc. This work is licensed under the Creative Commons Attribution International License (CC BY 4.0).

http://creativecommons.org/licenses/by/4.0/

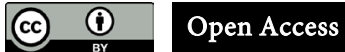

\begin{abstract}
To our knowledge this is the first report to provide a detailed description of surgical procedure for adhesiolysis and hepatectomy in patients who have undergone esophagectomy and reconstruction. We performed a hepatic resection of the left medial segment in a patient with a reconstructed stomach tube after esophagectomy for the esophageal carcinoma. The reconstructed stomach tube overlapped with the left medial segment of the liver and the hepatoduodenal ligament and was extensively and strongly adhered to them. It is important for clinicians to know how to perform the detachment procedure successfully in order to secure a surgical field for liver resection without damaging the fragile reconstructed gastric tube. In order to avoid vascular injury of the stomach tube, it was decided that detachment around the hepatoduodenal ligament preceded detachment of the stomach tube from the liver. After complete separation of the hepatoduodenal ligament from the stomach tube, the hepatoduodenal ligament was encircled with tape. Subsequently, adhesiolysis was performed between the stomach tube and the liver. Finally, parenchymal transection was performed using the intermittent hepatic inflow occlusion and crush clamping techniques to dissect the parenchyma. The patient was discharged two weeks after surgery without complication.
\end{abstract}

\section{Keywords}

Detachment of Adhesions, Liver Resection, Reconstructed Stomach Tube

\section{Introduction}

Due to the remarkable progress made in surgical techniques and outcomes, we 
increasingly encounter situations in which it is necessary to perform further major abdominal surgery (such as hepatectomy) in patients who have already undergone major digestive surgery [1]. Depending on the reconstruction carried out in the first major digestive surgery, the surgical field for the next major surgery is often extremely limited. Specifically, digestive organs reconstructed in past surgeries are fragile and easily damaged during adhesiolysis in the second major abdominal surgery.

We performed a successful adhesiolysis followed by hepatic resection of the left medial segment in a patient with a reconstructed stomach tube after esophagectomy for the esophageal carcinoma, without damaging the fragile stomach tube. This procedure was challenging as the reconstructed stomach tube overlapped with the left medial segment of the liver and the hepatoduodenal ligament and was extensively and strongly adhered to these structures.

It is important for clinicians to know how to perform a detachment procedure to secure the surgical field for liver resection without damaging the fragile reconstructed gastric tube [2]. Hence, the aim of this paper is to present a safe technique for detaching the gastric tube from the liver surface and the hepatoduodenal ligament in order to perform hepatectomy. We herein report on the ingenuity of our surgical procedures in hepatectomy after esophageal reconstruction.

\section{Case Presentation}

The patient was a 70-year-old Japanese male with a previous history of left lateral sectionectomy for intrahepatic cholangiocarcinoma (ICC) and esophagectomy for coexisting esophageal carcinoma about one month after the hepatectomy. Fifteen months after the esophagectomy, intrahepatic recurrence (diameter of $2.5 \mathrm{~cm}$ in S4) for ICC was detected. Re-hepatectomy was scheduled to address the intrahepatic recurrence. In response to previous esophageal reconstruction surgery, the reconstructed stomach tube had extensively and strongly adhered to peritoneum on the ventral side of the left medial segment of the liver as well as to the hepatoduodenal ligament (Figure 1). Blood supply to the gastric tube lay along the lesser and greater curvatures of the stomach tube and it was necessary to devise a method to remove adhesions without damaging blood flow to the stomach tube.

\section{Operative Procedures}

Adhesiolysis between the abdominal wall and the abdominal organs was performed through a J-shaped laparotomy incision. The procedure required great care due to extensive and severe adhesion after two previous abdominal surgeries. Particular attention was paid to adhesiolysis around the reconstructed gastric tube in order to avoid causing any injuries to vessels related to the reconstructed stomach, which overlaid the surgical field for hepatectomy. Blood flow originating from the right gastroepiploic artery and the right gastric artery, 
which were the only sources of blood supply preserved in creating the stomach tube, lay along the greater gastric curvature and the lesser gastric curvature.

In order to avoid vascular injury to the stomach tube, it was decided that detachment around the hepatoduodenal ligament preceded detachment of the gastric tube from the liver (Figure 2). In our institute, we routinely perform the Pringle maneuver during liver transection in order to reduce blood loss and preserve liver function in the early postoperative period [3]. Therefore, we needed to prepare the hepatoduodenal ligament for taping required by the Pringle maneuver. After adequately releasing the duodenum and the transverse colon from the surface of the liver, we created space on the right side of the hepatic duodenal ligament to allow a blunt dissector to easily pass through the foramen of Winslow.

Adhesiolysis of the dorsal side of the hepatoduodenal ligament was performed from the right side through the foramen of Winslow. After wrapping of the

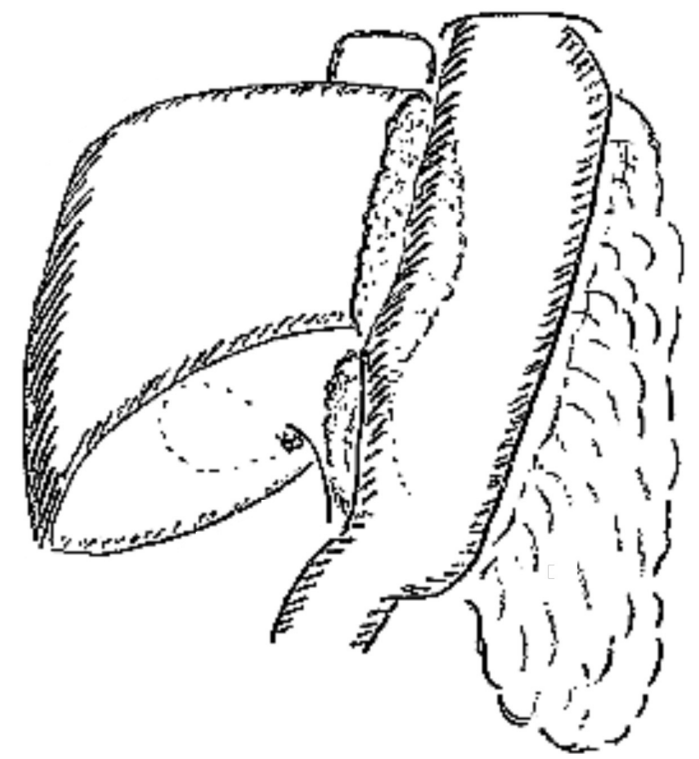

Figure 1. The stomach tube adhering to the liver and the hepatoduodenal ligament.

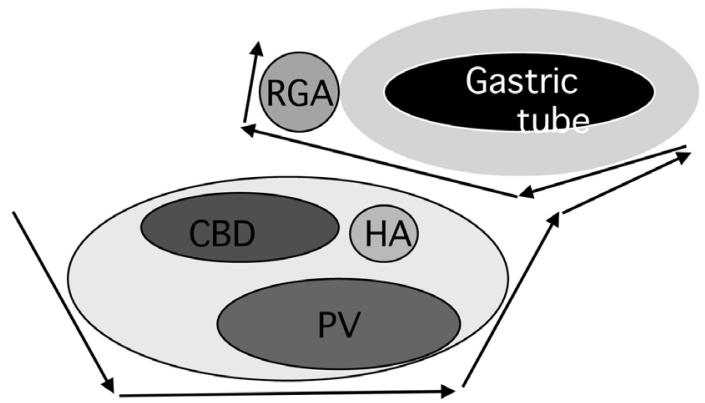

RGA, CBD, HA, and PV indicate right gastric artery, common bile duct, hepatic artery and portal vein, respectively.

Figure 2. Schema of a transverse image showing the positional relationship of the vessels and a bile duct. Adhesiolysis is performed according to the direction of the arrows. CBD, $\mathrm{HA}$ and PV are present in the hepatoduodenal ligament. 
dorsal side of the hepatoduodenal ligament, adhesiolysis of the left side of the hepatoduodenal ligament was safely performed. Final adhesiolysis was performed around the hepatoduodenal ligament between the dorsal side of the stomach tube and the ventral side of the hepatoduodenal ligament. Adhesion on the side of the lesser curvature of the stomach tube was tight and strong, whereas there was room in the length of the greater curvature of the stomach tube, and, despite adhesion, the tension was not as strong in this area. Hence, we could easily perform adhesiolysis of the dorsal side of the stomach from the left side, while paying careful attention to the vessels on the greater curvature.

Complete separation was achieved between the hepatoduodenal ligament and the stomach tube, and the hepatoduodenal ligament was encircled with tape.

Subsequently, adhesiolysis was performed between the stomach tube and the liver. After release, the stomach tube was also encircled with tape and was drawn to the left. After complete adhesion detachment between the liver and other organs, the medial section of the liver and the cut end of the previous resection (segments 2 and 3) were clearly exposed (Figure 3). The right transection line (Cantlie line) lay between segments 4 and right lobe, where the middle hepatic vein was exposed and preserved. Finally, parenchymal transection was performed. We used the intermittent hepatic inflow occlusion and crush clamping techniques to dissect the parenchyma. Total blood loss was $1660 \mathrm{ml}$ and surgery lasted 6 hours and 47 minutes. The patient was discharged two weeks after the operation without complication.

After the hepatectomy described in this paper, the patient received re-re-hepatectomy for intrahepatic re-recurrence, percutaneous radiofrequency ablation for intrahepatic re-re-recurrence, and re-re-re-hepatectomy for intrahepatic re-re-re-recurrence. There was no sign of recurrence of concerning

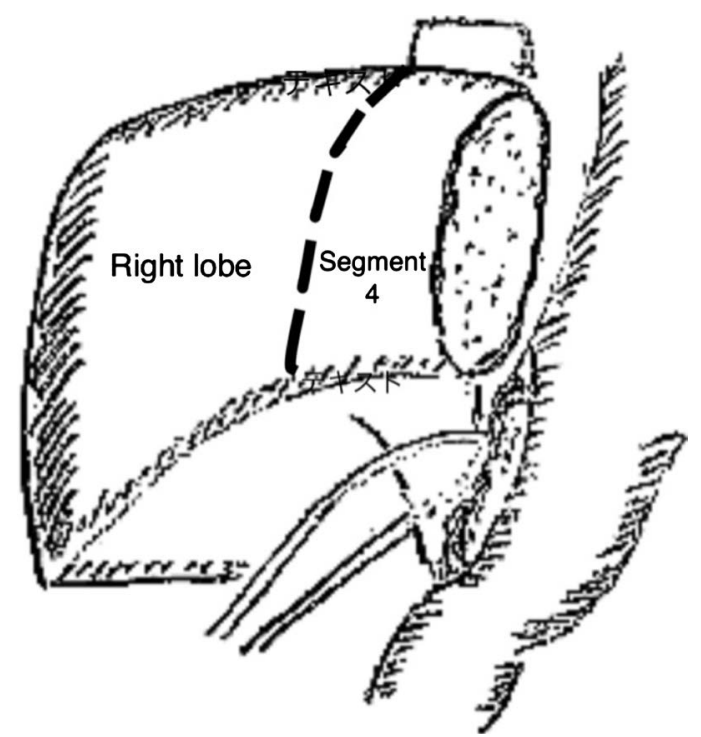

Figure 3. The hepatoduodenal ligament is encircled with the stomach tube pulled to the left. The broken line shows the right transection line (Cantlie line) which lies between segments 4 and right hepatic lobe. 
esophageal carcinoma [4]. The patient died survived 44 months after the surgery described here and died of cancer recurrence.

\section{Discussion}

Little has been published concerning liver resection after reconstruction of the esophagus, and few papers mention the salient points surrounding these surgeries [1] [2] [5].

Most notably, Takayama et al. reported that during surgery, the specific concern was to avoid any injuries to vessels related to the tubed stomach overlying the hepatectomy field [2]. It should be technically difficult to perform hepatectomy after surgery for esophageal cancer, but details of the surgical procedure have not been described in previous reports [1] [5].

In order to perform the surgery safely, it is necessary to first confirm the nature and extent of adhesiolysis required in order to adequately prepare the surgical field.

Hepatic pedicle clamping (the Pringle maneuver), which interrupts the arterial and portal venous inflow to the liver, is a standard procedure in hepatic surgery [3]. We routinely use the Pringle method to safely perform hepatectomy. However, in order to successfully perform the Pringle maneuver, it is necessary to wrap the hepatoduodenal ligament. Prior to this procedure, it is necessary to safely remove all adhesion between the gastric tube and the hepatoduodenal ligament, and then to carefully remove the adhered organs from the liver.

The reconstructed gastric tube is a fragile organ with surrounding feeding vessels and partial tension. In this case, hepatectomy could be safely performed without damaging the gastric tube. Even after performing major surgery such as esophagectomy, in this manuscript, it was shown that the next major surgery can be performed if the adhesion detachment is properly performed. The aim of this paper is to provide evidence of successful adhesiolysis without damage to the gastric tube and to offer details of how this might be achieved in future cases.

\section{Funding}

No financial.

\section{Ethical Approval}

Ethical approval was not required and patients identifying knowledge were not presented in this report.

\section{Consent}

Informed consent was obtained from the patients for publication of this case report and accompanying images.

\section{Conflicts of Interest}

The authors declare no conflicts of interest regarding the publication of this paper. 


\section{References}

[1] Nanashima, A., Hiyoshi, M., Imamura, N., Yano, K., Hamada, T., Wada, T., Nishida, T., Tsuchiya, K., Kawano, F., Ikeda, T. and Takeno S. (2018) A Cohort Study on The Risk of Hepatectomy and Pancreatectomy after History of Abdominal Surgery on Other Organs. Annals of Hepato-Biliary-Pancreatic Surgery, 22, 344-349. https://doi.org/10.14701/ahbps.2018.22.4.344

[2] Takayama, T., Kato, H., Tachimori, Y., Watanabe, H., Furukawa, H., Takayasu, K., Sakamoto, M. and Makuuchi, M. (1996) Treatment of Rupture of a Liver Metastasis from Esophageal Leiomyosarcoma. Japanese Journal of Clinical Oncology, 26, 248-251. https://doi.org/10.1093/oxfordjournals.jjco.a023223

[3] Man, K.I., Fan, S.T., Ng, I.O., Lo, C.M., Liu, C.L. and Wong, J. (1997) Prospective Evaluation of Pringle Maneuver in Hepatectomy for Liver Tumors by a Randomized Study. Annals of Surgery, 226, 704-711. https://doi.org/10.1097/00000658-199712000-00007

[4] Fujisaki, S., Takashina, M., Tomita, R. and Takayama, T. (2007) A Case of Successful Management of Repeated Recurrent Intrahepatic Cholangiocarcinoma by Repeated Hepatectomy and Radiofrequency Ablations. Gan to Kagaku Ryoho, 34, 2105-2107.

[5] Iitaka, D., Shiozaki, A., Fujiwara, H., Ichikawa, D., Okamoto, K., Komatsu, S., Murayama, Y., Ikoma, H., Kuriu, Y., Nakanishi, M., Ochiai, T., Kokuba, Y., Sonoyama, T. and Otsuji E. (2013) A Case Involving Long-Term Survival after Esophageal Cancer with Liver and Lung Metastases Treated by Multidisciplinary Therapy: Report of a Case. Surgery Today, 43, 556-561.

https://doi.org/10.1007/s00595-012-0240-7 